Bio - grafia. Escritos sobre la Biología y su Enseñanza. ISSN 2027

Edición Extraordinaria. p.p. 1692-1700

Memorias del VIII Encuentro Nacional de Experiencias en Enseñanza de la Biología y la Educación Ambiental. III Congreso Nacional de Investigación en Enseñanza de la Biología.

\title{
CONCEPCIONES SOBRE EL SISTEMA INMUNOLÓGICO QUE POSEEN LOS ESTUDIANTES DE OCTAVO GRADO EN LA INSTITUCIÓN EDUCATIVA INEM "JULIÁN MOTTA SALAS" EN LA CIUDAD DE NEIVA-HUILA.
}

\author{
IMMUNE SYSTEM CONCEPTIONS IN EIGHTH GRADE STUDENTS IN THE \\ INSTITUTION INEM "JULIAN MOTTA SALAS" IN THE CITY OF NEIVA-HUILA.
}

\author{
Diego Reinaldo Culman Mendoza*, Yuri Lizeth Huependo Romero*, Elías Francisco \\ Amórtegui Cedeño, Sonia Echeverry Hernández \\ *diegoreinaldoyandrea@hotmail.com, ${ }^{*}$ yuripary11@gmail.com
}

Universidad Surcolombiana, Programa de Licenciatura en Ciencias Naturales: Física, Química y Biología, Semillero de investigación ENCINA - Semillero de investigación VIRHOBAC

\section{RESUMEN}

En esta investigación se describe las concepciones que poseen los estudiantes de octavo grado de la Institución Educativa INEM "Julián Motta Salas" de Neiva (HuilaColombia), sobre el tema de Sistema Inmunológico. Por su naturaleza el estudio se orientó como una investigación cualitativa y los instrumentos utilizados para la recolección de datos fueron un cuestionario y la observación participante durante el 2014-II. Los resultados mostraron que los estudiantes identifican variedad de concepciones frente al tema, y a partir de la labor docente con la ayuda del diseño e implementación de una unidad didáctica comprendida por 7 subtemáticas del sistema inmunológico, las cuales se abarcaron con el desarrollo de una práctica de laboratorio, una salida de campo y siete actividades de complemento que fueron desarrolladas en 10 sesiones de clases presenciales, permitieron la enseñanza-aprendizaje de diferentes contenidos conceptuales, procedimentales y actitudinales que favoreció la construcción de explicaciones de manera científica sobre el sistema inmunológico. De manera general, las concepciones de algunos de los estudiantes se mantuvieron durante el proceso formativo, para otros se observó algunas modificaciones, las cuales están relacionadas con las actividades formativas que se desarrollaron a través de la planificación e implementación de la unidad didáctica guiadas a través de las discusiones que se generaban en las sesiones de clase.

Palabras clave: concepciones, sistema inmunológico, unidad didáctica. 
Bio - grafia. Escritos sobre la Biología y su Enseñanza. ISSN 2027

Edición Extraordinaria. p.p. 1692-1700

Memorias del VIII Encuentro Nacional de Experiencias en Enseñanza de la Biología y la Educación Ambiental. III Congreso Nacional de Investigación en Enseñanza de la Biología.

\begin{abstract}
In this research the concepts that have the eighth graders of School INEM "Motta Julian Salas" of Neiva (Huila-Colombia), on the subject of Immune System described. By its nature the study was oriented as a qualitative research and the instruments used for data collection were a questionnaire and participant observation during the 2014-II. The results showed that students identify variety of views towards the subject, and from the teaching with the help of the design and implementation of a teaching unit comprised of seven sub-themes of the immune system, which is covered with the development of a practice of laboratory, a field-seven activities complement that were developed in 10 sessions of classes, allowed the teaching and learning of different conceptual, procedural and attitudinal contents which favored the construction of scientific explanations of how the immune system. In general, the views of some of the students were maintained during the training process, other modifications were observed, which are related to the training activities developed through the planning and implementation of the teaching unit guided through of the discussions that were generated in the class sessions.
\end{abstract}

KEY WORDS: conceptions, immune system, teaching unit.

\title{
INTRODUCCIÓN
}

En la Institución Educativa INEM "Julián Motta Salas" de la ciudad de Neiva-Huila poco se ha desarrollado el proceso investigativo; ya que a pesar de que es un elemento importante a la hora de construir nuevas concepciones científicas al estudiantado, los docentes no lo desarrollan debido a la carga académica que se les asigna o quizás a la falta de interés por esta.

Dentro de lo investigado se observó pocas evidencias a nivel local de proyectos sobre el cuerpo humano y específicamente en el campo de la inmunidad, lo cual es un componente del diseño curricular que se debe aplicar para el grado octavo. Es muy oportuno aclarar que a nivel de la Universidad Surcolombiana en el programa de Licenciatura en Ciencias Naturales: Física, Química y Biología no se presenta evidencias sobre trabajos de grado en concepciones del cuerpo humano y menos sobre el sistema inmunológico. 
Bio - grafia. Escritos sobre la Biología y su Enseñanza. ISSN 2027

Edición Extraordinaria. p.p. 1692-1700

Memorias del VIII Encuentro Nacional de Experiencias en Enseñanza de la Biología y la Educación Ambiental. III Congreso Nacional de Investigación en Enseñanza de la Biología.

Con base a lo anterior surge la siguiente pregunta de investigación la cual consiste en ¿Cómo favorece al proceso de enseñanza-aprendizaje del sistema inmunológico la implementación de una unidad didáctica en estudiantes de octavo grado en la Institución Educativa INEM "Julián Motta Salas" de la ciudad de Neiva- Huila?

\section{METODOLOGÍA}

La investigación estuvo diseñada bajo un enfoque cualitativo; se empleó el método de análisis de contenido que se considera como un proceso de codificación de contenido de un texto proporcionado por los participantes, y finalmente las técnicas de recolección de información utilizadas fueron la observación participante y un cuestionario que se aplicó tanto al inicio como al final del proceso formativo.

Una vez recogida la información con los diferentes instrumentos, previamente validados por expertos, se identificaron las oraciones con sentido, dadas por los estudiantes en el campo de la inmunología. Para el proceso de categorización se empleó el software Atlas.ti.

\section{RESULTADOS Y DISCUSIÓN}

A continuación se presenta los resultados iniciales de la investigación, que corresponden a las concepciones empleadas por los estudiantes para referirse al sistema inmunológico; cuya información se obtuvo del cuestionario diagnostico "Reconociendo Nuestros Guardianes de protección" antes de la intervención didáctica. Posteriormente, se precisa los principales obstáculos identificados en los estudiantes en función del aprendizaje de conceptos relacionados con el sistema inmunológico. Por último, se expone las concepciones finales elaboradas a partir de las respuestas dadas por ellos después de la intervención didáctica.

\section{Cuestionario Inicial}

El análisis de las oraciones acerca del sistema inmunológico con el propósito de identificar las categorías a las cuales se refieren los estudiantes, arrojo unas subcategorías como: vulnerabilidad y salud (ver figura 1). Posteriormente se presentan agrupaciones y tendencias obtenidas a partir de cada una de estas. 
Bio - grafia. Escritos sobre la Biología y su Enseñanza. ISSN 2027

Edición Extraordinaria. p.p. 1692-1700

Memorias del VIII Encuentro Nacional de Experiencias en Enseñanza de la Biología y la Educación Ambiental. III Congreso Nacional de Investigación en Enseñanza de la Biología.

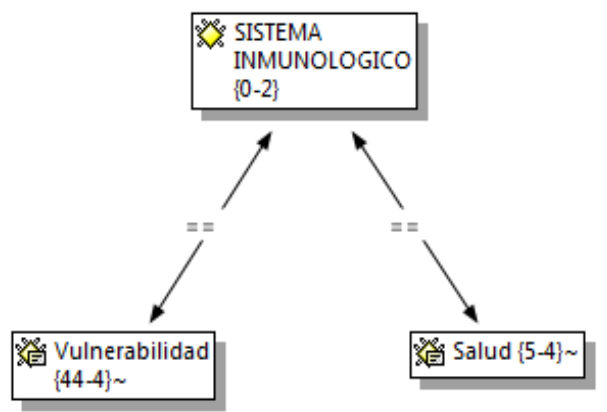

Figura 1. Subcategorías acerca de las concepciones de los estudiantes sobre sistema inmunológico en el cuestionario inicial.

Con relación a la subcategoría Vulnerabilidad que tiene el sistema inmunológico se encontró tres agrupaciones (enfermedad, lugares de contagio y medio de contagio) frente a las respuestas de los estudiantes como lo muestra la (ver figura 2).

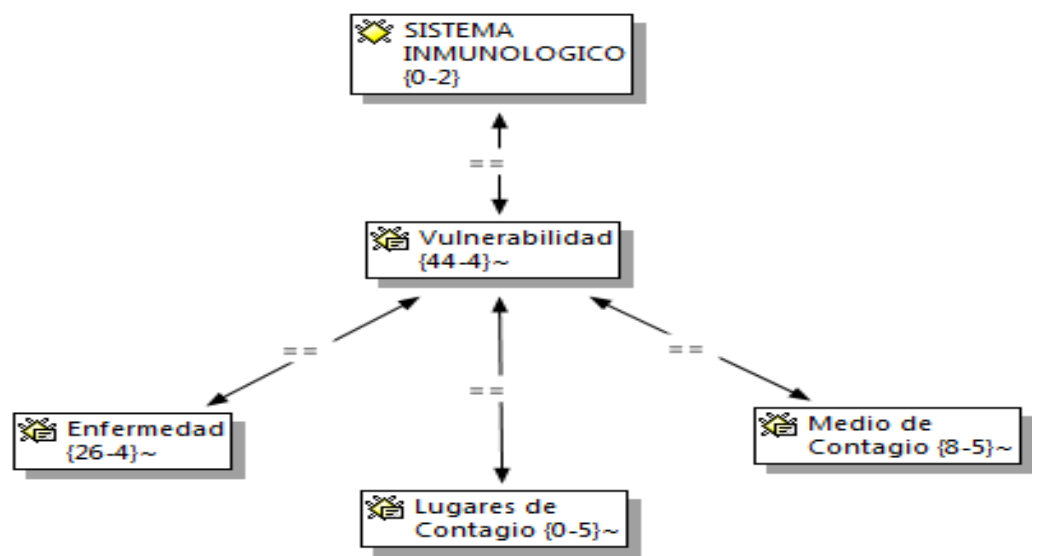

Figura 2. Agrupaciones sobre la Vulnerabilidad del sistema inmunológico halladas en el cuestionario inicial.

En los estudiantes se encuentra una alta existencia de explicaciones a nivel macroscópico sobre lugares de contagio de enfermedades, por ejemplo cuando especifican que ciertos lugares de Neiva como la plaza de "Sur abasto", hospitales, ríos, entre otros pueden ser lugares de infección, ya que éstos presentan grandes cantidades de suciedad, mugre, basuras, residuos sólidos no clasificados, aguas estancadas, entre otros. Por lo tanto se puede inferir que no existen explicaciones a nivel microscópico, por ejemplo para 
Bio - grafia. Escritos sobre la Biología y su Enseñanza. ISSN 2027

Edición Extraordinaria. p.p. 1692-1700

Memorias del VIII Encuentro Nacional de Experiencias en Enseñanza de la Biología y la Educación Ambiental. III Congreso Nacional de Investigación en Enseñanza de la Biología.

los estudiantes la presencia de microorganismos y la formación de colonias bacterianas en ciertos lugares, no son consideradas en términos de poblaciones. Además las características biológicas de éstos que son principalmente su mecanismo de reproducción, sus requerimientos nutricionales, características celulares y la forma en que se presenta en los lugares, tampoco son percibidas por los estudiantes, reafirmando los planteamientos de Jiménez (2003) quien sostiene que algunas de las dificultades del aprendizaje de la Biología en la educación secundaria están relacionadas con el hecho de que los estudiantes no atribuyen el concepto de célula a todos los organismos vivos, suelen identificar de los microorganismos tan solo que son "perjudiciales", poseen confusión sobre el papel de los microorganismos para el ser humano y la distinción funcional entre bacterias y virus.

Por último, se destaca que las concepciones de los estudiantes se alejan bastante del conocimiento científico y en su mayoría están nutridas, enriquecidas o favorecidas por el conocimiento cotidiano o el saber común y/o popular de la región exclusivamente.

Con relación a la subcategoría de Salud del sistema inmunológico se halló tres agrupaciones las cuales se pueden relacionar dependiendo de las respuestas que presentan los estudiantes (ver figura 3). Además partiendo de las respuestas se puede inferir que esta se funda en la noción de desequilibrio entre un conjunto de cualidades de carácter dual y complementario. Otros le dan una connotación de proceso y no de estado.

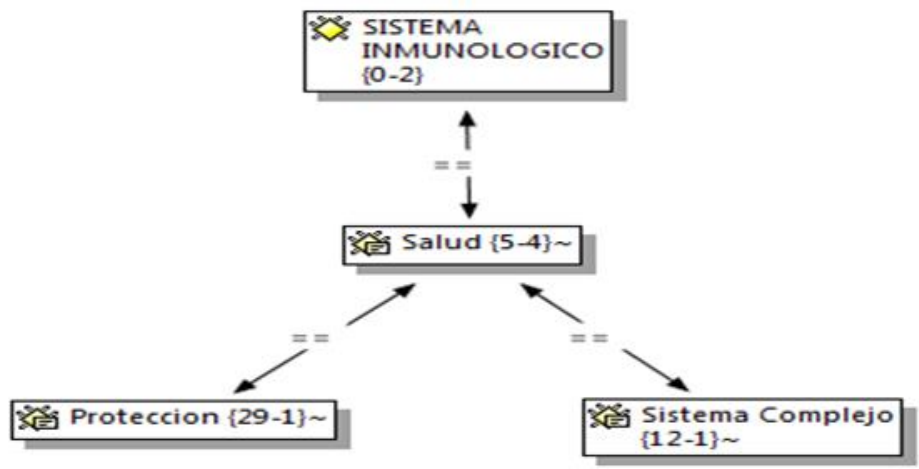

Figura 3. Agrupaciones sobre la Salud del sistema inmunológico halladas en el cuestionario inicial.

En el análisis de la agrupación de Salud se puede concluir que los estudiantes dan sus explicaciones haciendo uso del conocimiento científico; por ejemplo manifiestan que las 
Bio - grafia. Escritos sobre la Biología y su Enseñanza. ISSN 2027

Edición Extraordinaria. p.p. 1692-1700

Memorias del VIII Encuentro Nacional de Experiencias en Enseñanza de la Biología y la Educación Ambiental. III Congreso Nacional de Investigación en Enseñanza de la Biología.

primeras defensas recibidas son originadas por la leche materna y que a medida en que se va creciendo, estas también aumentando y crecen. Según Mere \& Pareja (1997) el niño recién nacido recibe lactancia materna exclusiva que permite establecer una flora exógena y endógena adecuada para el primer semestre de vida.

Los análisis anteriores permiten identificar los siguientes obstáculos frente al aprendizaje del sistema inmunológico:

> Privilegio del uso de lenguajes cotidianos para referirse a fenómenos relacionados con la inmunidad.

> Realización de explicaciones de carácter macroscópico de los fenómenos relacionados con la inmunidad, con el consecuente distanciamiento de explicaciones científicas que se constituirían en un indicador del aprendizaje profundo del fenómeno estudiado por parte de los estudiantes.

\section{Cuestionario Final}

Los hallazgos del cuestionario final permitieron establecer tres grandes subcategorías (vulnerabilidad, salud y mecanismos de defensas) sobre las concepciones de los estudiantes acerca del Sistema Inmunológico en el cuestionario final (ver figura 4).

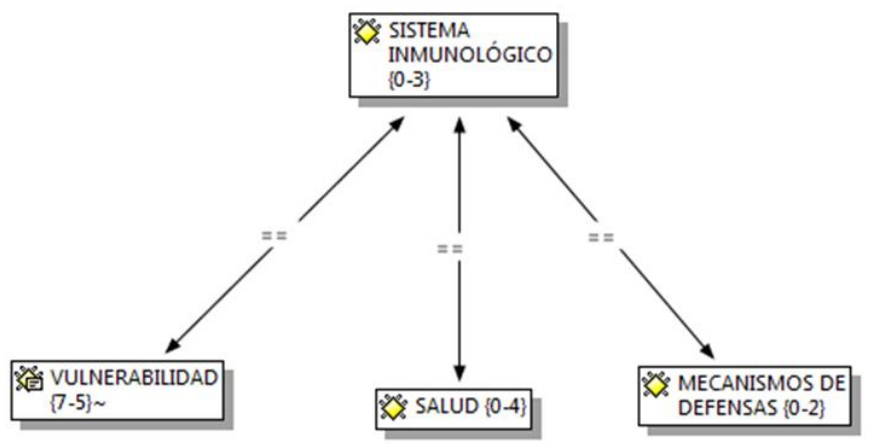

Figura 4. Subcategorías acerca de las concepciones de los estudiantes sobre sistema inmunológico en el cuestionario final. 
Bio - grafia. Escritos sobre la Biología y su Enseñanza. ISSN 2027

Edición Extraordinaria. p.p. 1692-1700

Memorias del VIII Encuentro Nacional de Experiencias en Enseñanza de la Biología y la Educación Ambiental. III Congreso Nacional de Investigación en Enseñanza de la Biología.

Con relación a la subcategoría Vulnerabilidad que tiene el sistema inmunológico se encontró cuatro agrupaciones (medio de contagio, lugares de contagio, enfermedad y agentes patógenos) frente a las respuestas de los estudiantes (ver figura 5).

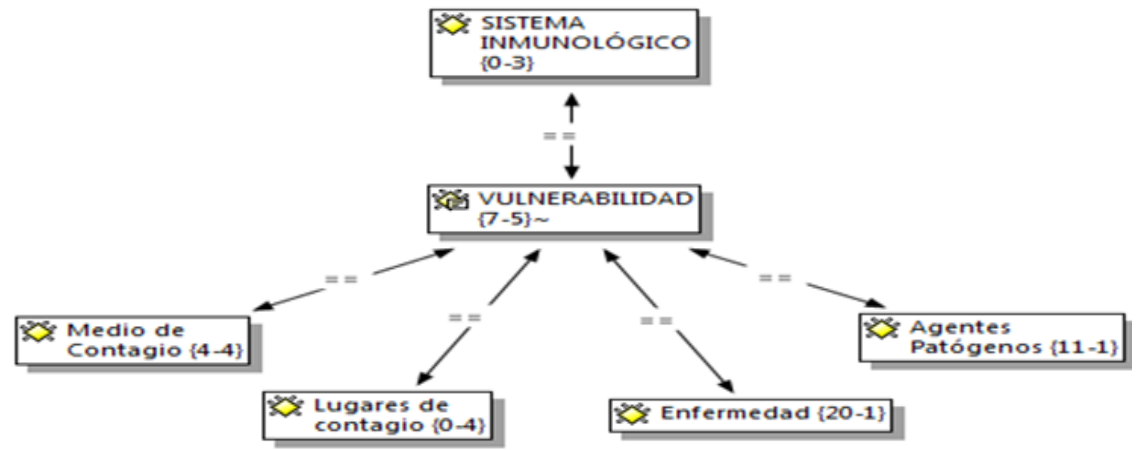

Figura 5. Agrupaciones sobre la Vulnerabilidad del sistema inmunológico halladas en el cuestionario Final.

En el análisis de las respuestas sobre la subcategoría Vulnerabilidad se concluye que, después de la intervención didáctica, los estudiantes mejoraron el uso del lenguaje especializado. Para dar las explicaciones, por ejemplo, ya no se refieren al mosquito Aedes aegyti con el término de "Zancudo", aunque no especifican que el mosquito hembra, infectado con el virus es el vector de transmisión de esta enfermedad al ser humano. Reconocen las enfermedades diferenciales causadas por bacterias y virus, como la tuberculosis, la gripe y el dengue; para este caso en particular los estudiantes identifican signos y síntomas de la enfermedad y la clasificación actual. Además deducen que estos microorganismos se pueden encontrar en lugares como sus casas y en la Institución Educativa INEM "Julián Motta Salas" de la ciudad de Neiva-Huila .Dicho de otra manera resuelven las preguntas más desde el conocimiento científico que desde el sentido común. En esta subcategoría, también se evidencio explicaciones de tipo científicas sobre el contagio de la gripe como producto del contacto directo entre el cuerpo de una persona y el de otra.

Con relación a la subcategoría Salud que tiene el sistema inmunológico se encontró tres agrupaciones (protección, sistema complejo, prevención) frente a las respuestas de los estudiantes (ver figura 6). 
Bio - grafia. Escritos sobre la Biología y su Enseñanza. ISSN 2027

Edición Extraordinaria. p.p. 1692-1700

Memorias del VIII Encuentro Nacional de Experiencias en Enseñanza de la Biología y la Educación Ambiental. III Congreso Nacional de Investigación en Enseñanza de la Biología.

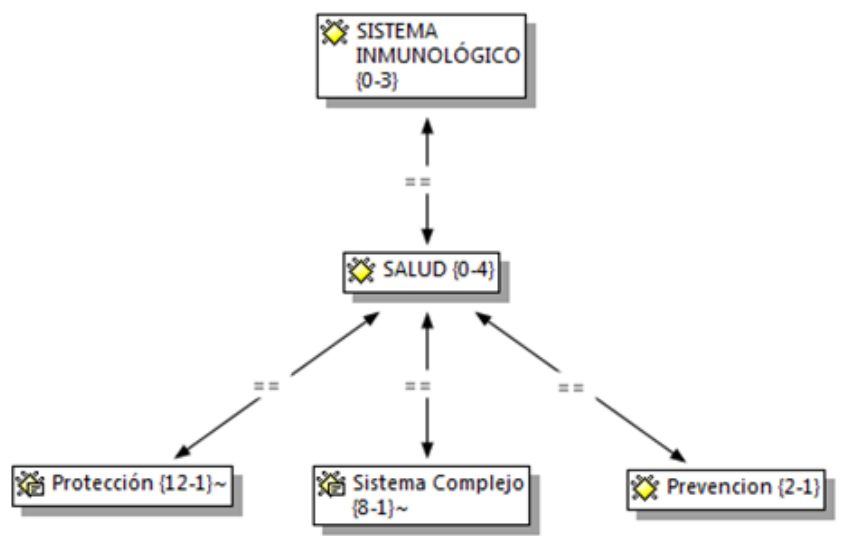

Figura 6. Agrupaciones sobre Salud del sistema inmunológico halladas en el cuestionario Final.

En el análisis de la información frente a la subcategoría de Salud se encontró explicaciones congruentes con el conocimiento científico como ocurre en la subcategoría anterior donde los estudiantes mejoraron el uso del lenguaje especializado, se refieren a algunos términos con mayor propiedad. Por ejemplo cuando hacen referencia al sistema inmunológico como una barrera protectora frente a los microorganismos causantes de enfermedades o de infecciones. Además reconocen la resistencia del cuerpo a las infecciones gracias a mecanismos de defensas distribuidos por todo el cuerpo y asociados con otros sistemas como el muscular, esquelético y digestivo.

Con relación a la subcategoría Mecanismos de defensas que tiene el sistema inmunológico se encontró una agrupación (sintomatología) frente a las respuestas de los estudiantes. Además se evidencio que los ellos concibe los síntomas de las distintas infecciones tratadas como resultado de la respuesta inmune del cuerpo humano.

\section{CONCLUSIONES}

De acuerdo con las concepciones iniciales de los estudiantes es importante resaltar que antes de la intervención didáctica los estudiantes presentaban un conocimiento más popular y cotidiano frente al sistema inmunológico, mientras que después de la implementación de la unidad didáctica estos ya hablaban desde un conocimiento más científico. 
Bio - grafia. Escritos sobre la Biología y su Enseñanza. ISSN 2027

Edición Extraordinaria. p.p. 1692-1700

Memorias del VIII Encuentro Nacional de Experiencias en Enseñanza de la Biología y la Educación Ambiental. III Congreso Nacional de Investigación en Enseñanza de la Biología.

Las subcategorías: vulnerabilidad y salud constituyen la columna vertebral del cuestionario inicial para explicar el sistema inmunológico, expresado por los estudiantes antes de la intervención didáctica. Después de la intervención didáctica las categorías que adquirieron mayor relevancia fueron: vulnerabilidad, salud y mecanismos de defensas las cuales se constituyen en la columna vertebral del cuestionario final para explicar el sistema inmunológico.

\section{BIBLIOGRAFIA}

Jiménez, M (2003). La enseñanza y el aprendizaje de la Biología. En: Jiménez, M; Caamaño, A; Oñorbe, A; Pedrinaci, E; Pro, A (2003). Enseñar Ciencias. Barcelona: Graó.

Meré, H.R \& Pareja, B.Z. (1997). Conocimientos sobre la lactancia materna que poseen los primigestas que acuden al control pre-natal del Hospital Nacional Docente Madre-Niño, San Bartolomé. Facultad de Medicina Humana, Universidad Nacional Mayor de San Marcos. Lima, Perú. 